\section{PARP-1 protein expression in glioblastoma multiforme}

\author{
A. Galia, ${ }^{1}$ A.E. Calogero, ${ }^{2}$ \\ R.A. Condorelli, ${ }^{2}$ F. Fraggetta, ${ }^{1}$ \\ C. La Corte, ${ }^{1}$ F. Ridolfo, ${ }^{3}$ P. Bosco, ${ }^{4}$ \\ R. Castiglione, ${ }^{2}$ M. Salemi ${ }^{4}$ \\ 'Unit of Pathology, Cannizzaro Hospital, \\ Catania; \\ ${ }^{2}$ Section of Endocrinology, Andrology and \\ Internal Medicine, Department of Internal \\ Medicine and Systemic Diseases, \\ University of Catania; \\ ${ }^{3}$ Post-graduate School in Clinical \\ Biochemistry, University of Milan; \\ ${ }^{4}$ Laboratory of Cytogenetics, Oasi \\ Institute for Research on Mental \\ Retardation and Brain Aging, Troina, Italy
}

\section{Abstract}

One of the most common type of primary brain tumors in adults is the glioblastoma multiforme (GBM) (World Health Organization grade IV astrocytoma). It is the most common malignant and aggressive form of glioma and it is among the most lethal ones. Poly (ADPribose) polymerase 1 (PARP-1) gene, located to $1 q 42$, plays an important role for the efficient maintenance of genome integrity. PARP-1 protein is required for the apoptosis-inducing factor (AIF) translocation from the mitochondria to the nucleus. PARP-1 is proteolytically cleaved at the onset of apoptosis by caspase-3. Microarray analysis of PARP-1 gene expression in more than 8000 samples revealed that PARP-1 is more highly expressed in several types of cancer compared with the equivalent normal tissues. Overall, the most differences in PARP-1 gene expression have been observed in breast, ovarian, endometrial, lung, and skin cancers, and non-Hodgkin's lymphoma. We evaluated the expression of PARP-1 protein in normal brain tissues and primary GBM by immunohistochemistry. Positive nuclear PARP-1 staining was found in all samples with GBM, but not in normal neurons from controls $(\mathrm{n}=4)$ and GBM patients $(\mathrm{n}=27)$. No cytoplasmic staining was observed in any sample. In conclusion, PARP-1 gene is expressed in GBM. This finding may be envisioned as an attempt to trigger apoptosis in this tumor, as well as in many other malignancies. The presence of the protein exclusively at the nucleus further support the function played by this gene in genome integrity maintenance and apoptosis. Finally, PARP-1 staining may be used as GBM cell marker.

\section{Introduction}

Glioblastoma multiforme (GBM), or World Health Organization (WHO) grade IV astrocytoma, is the most common type of primary brain tumor in adults. In addition, it is the most malignant and aggressive form of glioma and among the most lethal ones because of its highly invasive potential and least effective treatment available among solid tumors. ${ }^{1}$ Various genetic alterations lead to the development of malignant phenotype expression in human GBM. ${ }^{2,3}$ Heterogeneous pathological features associated with complex molecular heterogeneity are the major problems for the development of effective drug targets. ${ }^{4,5}$

GBMs can arise either de novo (primary) or progress to GBM from low grade gliomas (secondary) with two distinct molecular pathways. ${ }^{3}$ Significant indicators of anaplasia in gliomas include: atypia (coarse nuclear chromatin, nuclear pleomorphism, multinucleation), mitotic activity, cellularity, microvascular proliferation, and necrosis. Necrosis with the microvascular or endothelial cell proliferations is a histological hallmark of glioblastoma. ${ }^{3-5}$

Poly (ADP-ribose) polymerase 1 (PARP-1) gene, located to 1q42, is $43 \mathrm{~kb}-$ long and divided into 23 exons (OMIM 173870). Grube and Burkle ${ }^{6}$ suggested that a higher PARP-1 action capacity may contribute to an efficient maintenance of genome integrity. Yu et al..$^{7}$ showed that PARP-1 activation is required for translocation of apoptosis-inducing factor (AIF) from the mitochondria to the nucleus. PARP-1 protein is then proteolytically cleaved at the onset of apoptosis by caspase- $3 ;{ }^{8}$ furthermore, PARP1 activity and poly (ADP-ribose) (PAR) polymer, mediate PARP-1-induced cell death. ${ }^{8,9}$

Genetic and pharmacological studies found that overexpression of PARP-1 is a key mediator of programmed-necrotic cell death in vivo. In addition, PARP-1 appears to be also involved in programmed cell death processes, such as apoptosis or macroautophagocytotic cell death. ${ }^{10}$ Microarray analysis showed that PARP-1 mRNA expression in histologically normal tissues is low and uniform across a wide variety of tissues. ${ }^{11}$ Furthermore, microarray analysis of PARP-1 gene expression in more than 8000 samples revealed that PARP-1 is more highly expressed in several types of tumors compared with the equivalent normal tissue. Overall, the most striking differences in PARP-1 gene expression have been observed in breast, ovarian, endometrial, lung, skin cancers, and non-Hodgkin's lymphoma. ${ }^{11}$

The present study was undertaken to evaluate the expression of PARP-1 protein in normal brain tissues and primary glioblastomas (grade IV WHO) with immunohistochemistry.
Correspondence: Prof. Aldo E. Calogero, Section of Endocrinology, Andrology and Internal Medicine, Department of Internal Medicine and Systemic Diseases, University of Catania, via S. Sofia 78, 95123 Catania, Italy.

E-mail: acaloger@unict.it

Key words: Glioblastoma multiforme, brain, immunohistochemistry, PARP-1 gene.

Received for publication: 26 November 2011. Accepted for publication: 26 January 2012

This work is licensed under a Creative Commons Attribution NonCommercial 3.0 License (CC BYNC 3.0).

(C) Copyright A. Galia et al., 2012

Licensee PAGEPress, Italy

European Journal of Histochemistry 2012; 56:e9 doi:10.4081/ejh.2012.e

\section{Materials and Methods}

\section{Patient and control brain samples}

The comparison has been made between: i) 4 normal tissues brain removed with post mortem autopsy by normal donors (2 males and 2 females); ii) 27 non-tumor peripheral tissues brain removed during surgical resection on patients with GBM and 27 tumor tissues WHO grade IV GBM, specifically, by 14 male patients ( $58.3 \pm 10.0$ years) and 13 female patients $(58.3 \pm 12.6$ years) (Table 1$)$.

Diagnoses of GBM were made on histological sections stained with hematoxylin-eosin and the sections were evaluated by at least two operators. The protocol was approved by the internal Institutional Review Board and an informed written consent was obtained from each patient with GBM or, if deceased, by his/her relatives.

\section{Immunohistochemical staining}

Brain sections ( $4 \mu \mathrm{m}$ thick) were obtained from all normal donors and GMB patients. Brain sections from GBM patients included both GBM and normal tissue (Table 1). All sections were formalin-fixed and paraffin-embedded following standard methods.

PARP-(F-2), a mouse monoclonal antibody raised against PARP-1 protein, was used for immunohistochemistry (Santa Cruz Biotechnology, Inc., Heidelberg, Germany) PARP-1 (F-2): sc-8007). As indicated by the manufacturer instructions, this antibody, at the dilution of 1:300, has been shown to reliably recognize PARP-1 proteins in glioblastomas and normal brain by immunohistochemistry. Slides were deparaffinized, rehydrated, subjected to three 5-min cycles in a microwave at $360 \mathrm{~W}$ in citrate buffer, preincubated in $3 \%$ 
$\mathrm{H}_{2} \mathrm{O}_{2}$ in citrate buffer, and thoroughly washed in $50 \mathrm{~mm}$ Tris-Cl (pH 7.4), $150 \mathrm{~mm} \mathrm{NaCl}$ Tris buffered saline (TBS) containing $0.05 \%$ Tween 20 (washing buffer). Slides were then preincubated with $3 \%$ bovine serum albumin (BSA) in TBS for 30 min, incubated with 1:300 dilution of anti-PARP-1 antibody in TBS containing $1 \% \mathrm{BSA}$, thoroughly washed in washing buffer before detection with the LSAB 2 kit (anti mouse, biotinylated and peroxidaselabeled streptavidin) and 3,3-diaminobenzidine-4HCl (DAB; Dako, Carpinteria, CA, USA) from Dako, following the instructions contained in the kit. After detection, the sections were counterstained with haematoxylin, dehydrated and mounted in xylen-based DPX mountant (BDH, Pool, UK). ${ }^{12}$

\section{Microscopic evaluation}

Slides were observed and cells visually scored at 20X and 40X. To evaluate the percent- age of positive tumor cells, microscopic fields were chosen such that non-tumor cells were also included. The fraction of PARP-1 positive cells was evaluated independently in a blinded fashion by two co-authors. No significant difference was observed between the two observers.

\section{Statistical analysis}

Results are expressed as mean \pm SEM. Data were analyzed by chi-square test and statistical analysis was performed with Graph Pad Prism 5 software (Graph Pad Software, Inc. La Jolla, CA, USA). The statistical significance was accepted for the p value lower than 0.05 .

\section{Results}

Normal tissues (4 autopsies and 27 non- tumoral tissue of the same subjects with brain GBM) did not show any nuclear or cytoplasmic signal for the protein PARP-1 (Table 1, Figure 1A). The expression of PARP-1 was also evaluated in 27 samples of GBM, PARP-1 positive nuclear signal was present in all 27 samples with the mean of nuclei involved of $87.03 \pm 5.04 \%(\mathrm{P}<0.005)$. No cytoplasmic staining was observed in the samples (Table 1 , Figure 1B). The signal was present on the nuclear membrane and nuclear chromatin.

\section{Discussion}

This study showed that PARP-1 protein is detectable in GBM cells, but is undetectable in normal brain tissue. The protein seems to be exclusively localized in the nucleus. This observation suggests that $P A R P-1$ may play a role in this tumor and that the presence of the

Table 1. Expression PARP-1 protein in WHO glioblastoma multiforme grade IV astrocytoma.

\begin{tabular}{|c|c|c|c|c|c|}
\hline & Sex & Age (years) & Brain localization & Histological diagnosis & Positive cell nuclei (\%) \\
\hline Case 1 & Male & 41 & Right parietal lobe & Glioblastoma IV WHO & 95 \\
\hline Case 2 & Male & 61 & Right temporal lobe & Glioblastoma IV WHO & 85 \\
\hline Case 3 & Male & 51 & Left temporal lobe & Glioblastoma IV WHO & 90 \\
\hline Case 4 & Male & 67 & Right temporal lobe & Glioblastoma IV WHO & 90 \\
\hline Case 5 & Male & 66 & Right frontal lobe & Glioblastoma IV WHO & 80 \\
\hline Case 6 & Male & 68 & Left parietal lobe & Glioblastoma IV WHO & 85 \\
\hline Case 7 & Male & 60 & Left frontal lobe & Glioblastoma IV WHO & 90 \\
\hline Case 8 & Male & 68 & Right frontal lobe & Glioblastoma IV WHO & 85 \\
\hline Case 9 & Male & 66 & Right frontal lobe & Glioblastoma IV WHO & 90 \\
\hline Case 10 & Male & 53 & Right parietal lobe & Glioblastoma IV WHO & 95 \\
\hline Case 11 & Male & 51 & Left temporal lobe & Glioblastoma IV WHO & 80 \\
\hline Case 12 & Male & 60 & Left frontal lobe & Glioblastoma IV WHO & 85 \\
\hline Case 13 & Male & 38 & Left occipital lobe & Glioblastoma IV WHO & 85 \\
\hline Case 14 & Male & 66 & Left occipital lobe & Glioblastoma IV WHO & 80 \\
\hline Case 15 & Female & 50 & Left frontal lobe & Glioblastoma IV WHO & 95 \\
\hline Case 16 & Female & 69 & Left frontal lobe & Glioblastoma IV WHO & 90 \\
\hline Case 17 & Female & 66 & Left frontal lobe & Glioblastoma IV WHO & 85 \\
\hline Case 18 & Female & 43 & Right temporal lobe & Glioblastoma IV WHO & 90 \\
\hline Case 19 & Female & 62 & Right temporal lobe & Glioblastoma IV WHO & 80 \\
\hline Case 20 & Female & 60 & Right frontal lobe & Glioblastoma IV WHO & 90 \\
\hline Case 21 & Female & 67 & Left frontal lobe & Glioblastoma IV WHO & 95 \\
\hline Case 22 & Female & 28 & Right temporal lobe & Glioblastoma IV WHO & 80 \\
\hline Case 23 & Female & 50 & Brain ventricle & Glioblastoma IV WHO & 85 \\
\hline Case 24 & Female & 75 & Right frontal lobe & Glioblastoma IV WHO & 90 \\
\hline Case 25 & Female & 62 & Left frontal lobe & Glioblastoma IV WHO & 80 \\
\hline Case 26 & Female & 60 & Left frontal lobe & Glioblastoma IV WHO & 90 \\
\hline Case 27 & Female & 66 & Left frontal lobe & Glioblastoma I VWHO & 85 \\
\hline Autopsy 1 & Male & 66 & Right frontal lobe & Normal & Not applicable \\
\hline Autopsy 2 & Male & 54 & Brain ventricle & Normal & Not applicable \\
\hline Autopsy 3 & Female & 41 & Right parietal lobe & Normal & Not applicable \\
\hline Autopsy 4 & Female & 70 & Right temporal lobe & Normal & Not applicable \\
\hline
\end{tabular}




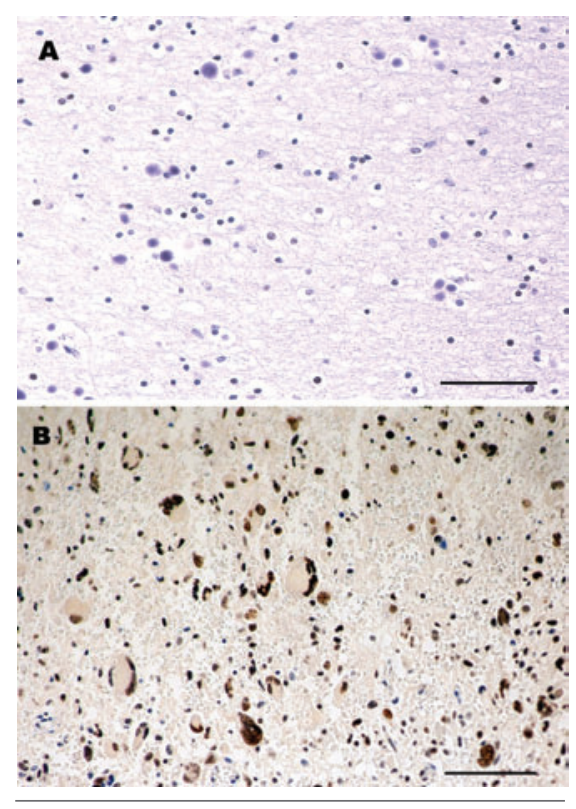

Figure 1. Immunohistochemistry of brain and glioblastoma multiforme IV WHO. A) Normal Brain, autopsy 3 (right parietal lobe); B) Glioblastoma multiforme IV WHO, case 1 (right parietal lobe); A and B hematoxylin counterstain; scale bar: 80 mm).

protein may be used as a cell marker. Indeed, previous studies $^{13,14}$ found the expression of PARP-1 in brain whit GBM, in normal brain and in nucleus and cytoplasm of human neurons, also Wharton et al. $2000^{15}$ have observed expression of PARP-1 in multicellular tumour spheroids, derived from human glioma cell lines.

PARP-1 gene plays a significant role in cell death both in cell necrosis and in apoptosis. Massive DNA damage that typically results from necrotic stimuli elicits a major increase of PARP-1 activity, which rapidly depletes the intracellular concentration of nicotine adenine dinucleotide $\left(\mathrm{NAD}^{+}\right)$and ATP. Increased consumption of ATP, in the effort to re-synthesize $\mathrm{NAD}^{+}$, leads to energy crisis culminating in cell death. ${ }^{16}$ This may in part justify the presence of PARP-1 protein in GBMs and in other tumors, such as ovarian, endometrial, lung, breast, skin cancers, and non-Hodgkin's lymphoma, ${ }^{11}$ as well as in a patients with Parkinson disease and multiple head and neck squamous cell in whom we recently reported an increased PARP-1 mRNA expression. ${ }^{17}$

The understanding of the mechanism that activates the expression of PARP-1 gene requires significant further work to characterize its inter-domain interactions and its DNAdependence, both at a structural and a functional level. ${ }^{18}$ Nonetheless, Eustermann and colleagues provided valuable insights into recognition of chromosomal DNA single-strand breaks, the crucial first step of the activation process, by PARP-1 DNA binding domain..$^{18}$ In fact, PARP-1 is a highly abundant chromatinassociated enzyme present in all higher eukaryotic cell nuclei, where it plays key roles in maintenance of genomic integrity, chromatin remodeling, and transcriptional control. PARP-1 gene product binds to DNA single- and double-strand breaks through an N-terminal region containing two zinc fingers, F1 and F2. The C-terminal catalytic domain of the PARP-1 protein is activated via an unknown mechanism, causing formation and addition of the poly (adenosine diphospho-ribose) complex to acceptor proteins including PARP-1 itself. ${ }^{19}$

Several reports from various laboratories indicate that inhibition or absence of PARP-1 provides remarkable protection in disease models, such as septic shock, diabetes, stroke, myocardial infarction, and ischemia, which are characterized predominantly by programmed-necrotic cell death. ${ }^{20}$ In addition, PARP-1 activation has been causally connected to photoreceptor cell death; ${ }^{21}$ this observation has more recently been confirmed by other authors, ${ }^{22}$ who showed a causal involvement of PARP-1 in retinal degeneration, a neurodegenerative diseases affecting photoreceptors and causing blindness in humans. Some authors, besides, observed that poly(ADP-ribose) polymerase-1 inhibitor, increases the antitumor activity against intracranial melanoma, glioma, lymphoma, hematological neoplasias; ${ }^{23-25}$ these data suggest the potential role that could have PARP-1 inhibitors in the therapy against glioblastoma.

Activation of PARP-1 in response to DNA damage is an important mechanism to keep homeostasis or to trigger apoptosis. The expression and function of PARP-1 has been studied in primary human lung cell cultures from normal human bronchial epithelial cells (NHBEC) and peripheral lung cells (PLC) from lung cancer patients grown as explant cultures over a period of 12 weeks. PARP-1 protein was expressed in all the cell culture derived from bronchial epithelium explants. ${ }^{26}$ Overall, recent data on PARP-1 agree on the meaning of this gene expression in tumors. Accordingly, the results of this study indicate that PARP-1 gene may play a role in GBMs.

In conclusion, PARP-1 protein (with this antibody) is detectable in the nuclei of all GBM cells, but is undetectable in normal cells. Given the role of PARP-1 protein in cell death (both necrosis and apoptosis) and in DNA repair mechanisms, it may be hypothesized that the presence of PARP-1 protein in the nucleus of the GBM is an attempt to trigger apoptosis in this type of tumor as well as in many other cancers. ${ }^{11,17}$ In the context of the tumor biology, this may be envisioned as a defense mechanism regardless of the outcome on the prognosis of patients with GBM. Finally, the evidence that PARP-1 positive staining is exclusively present within the nucleus adds further evidence for the above-mentioned role played by this protein.

\section{References}

1. Furnari FB, Fenton T, Bachoo RM, Mukasa A, Stommel JM, Stegh A et al. Malignant astrocytic glioma: genetics, biology, and paths to treatment. Genes Dev 2007;21:2683-10.

2. Lang FF, Miller DC, Koslow M, Newcomb EW. Pathways leading to glioblastoma multiforme: a molecular analysis of genetic alterations in 65 astrocytic tumors. J Neurosurg 1994;81:427-36.

3. Kleihues P, Ohgaki H. Primary and secondary glioblastomas: from concept to clinical diagnosis. Neuro-Oncol 1999;1:44-51.

4. Kleihues P, Burger PC, Scheithauer BW. The new WHO classification of brain tumors. Brain Pathol 1993;3:255-68.

5. Rasheed BK, Wiltshire RN, Bigner SH, Bigner DD. Molecular pathogenesis of malignant gliomas. Curr Opin Oncol 1999;11:162-67.

6. Grube K, Bürkle A. A Poly(ADP-ribose) polymerase activity in mononuclear leukocytes of 13 mammalian species correlates with species-specific life span. Proc Natl Acad Sci USA 1992;89:1175-63.

7. Yu SW, Wang H, Poitras MF, Coombs C, Bowers WJ, Federoff HJ. et al. Mediation of poly(ADP-ribose) polymerase-1-dependent cell death by apoptosis-inducing factor. Science 2002;297:259-63.

8. Andrabi SA, Kim NS, Yu SW. Poly(ADPribose) (PAR) polymer is a death signal.Proc Natl Acad Sci USA 2006;103:18308-13.

9. Yu SW, Andrabi SA, Wang H. Apoptosisinducing factor mediates poly (ADPribose) (PAR) polymer-induced cell death. Proc Natl Acad Sci USA 2006;103:18314-9.

10. Hassa PO, Hottiger MO. The diverse biological roles of mammalian PARPs, a small but powerful family of poly-ADP-ribose polymerases. Front Biosci 2008;13:304682.

11. Ossovskaya V, Koo IC, Kaldjian EP, Alvares C, Sherman BM. Upregulation of poly(ADP-ribose) polymerase-1 (PARP1) in triple-negative breast cancer and other primary human tumor types. Genes Cancer 2010;8:812-21.

12. Salemi M, Calogero AE, Zaccarello G, Castiglione R, Cosentino A, Campagna C. et al. Expression of SPANX proteins in normal prostatic tissue and in prostate cancer. Eur J Histochem 2010;54:e41.

13. Kase M, Vardja M, Lipping A, Asser T, Jaal 
J. Impact of PARP-1 and DNA-PK expression on survival in patients with glioblastoma multiforme. Radiother Oncol 2011;101:127-31.

14. Cookson MR, Ince PG, Usher PA, Shaw PJ. Poly(ADP-ribose) polymerase is found in both the nucleus and cytoplasm of human CNS neurons. Brain Res 1999;834:182-5.

15. Wharton SB, McNelis U, Bell HS, Whittle IR. Expression of poly(ADP-ribose) polymerase and of poly(ADP-ribosyl)ation in glioblastoma and in a glioma multicellular tumour spheroid model. Neuropathol Appl Neurobiol 2000;26:528-35.

16. Ha HC, Snyder SH. Poly (ADP-ribose) polymerase is a mediator of necrotic cell death by ATP depletion. Proc Natl Acad Sci USA 1999;96:13978-82.

17. Calogero AE, Soma PF, Giuffrida MC, Giuffrida D, La Vignera S, Romano C, et al. PARP1 and CASP3 gene expression in a patient with multiple head and neck squamous cell carcinoma and Parkinson disease. Hum Cell. 2011 [Epub ahead of print].

18. Eustermann S, Videler H, Yang JC, Cole
PT, Gruszka D, Veprintsev D, Neuhaus D. The DNA-binding domain of human PARP1 interacts with DNA single-strand breaks as a monomer through its second zinc finger. J Mol Biol 2011; 407:149-70.

19. Kannan S, Fang W, Song G, Mullighan CG, Hammitt R, McMurray J. et al. Notch/HES1-mediated PARP1 activation: a cell-type specific mechanism for tumor suppression. Blood 2011;117:2891-900.

20. Schreiber V, Dantzer F, Ame JC, de Murcia G. Poly(ADP-ribose): novel functions for an old molecule. Nat Rev Mol Cell Biol 2006;7:517-28.

21. Paquet-Durand F, Silva J, Talukdar T, Jonhnson LE, Azadi S, van Veen T, et al. Excessive activation of poly (ADP-ribose) ploymerase contributes to inherited photoreceptor degeneration in the retinal degeneration 1 mouse. The Journal of Neuroscience 2007;27:10311-9.

22. Sahaboglu A, Tanimoto N, Kaur J, SanchoPelluz J, Huber G, Fahl E, et al. PARP1 gene knock-out increases resistance to retinal degeneration without affecting retinal function. PLoS One 2010;5:e15495.
23. Tentori L, Leonetti C, Scarsella M, D'Amati G, Vergati M, Portarena I, et al. Systemic administration of GPI 15427, a novel poly(ADP-ribose) polymerase-1 inhibitor, increases the antitumor activity of temozolomide against intracranial melanoma, glioma, lymphoma. Clin Cancer Res 2003; 9:5370-9.

24. Russo AL, Kwon HC, Burgan WE, Carter D, Beam K, Weizheng X, et al. In vitro and in vivo radiosensitization of glioblastoma cells by the poly (ADP-ribose) polymerase inhibitor E7016. Clin Cancer Res 2009;15: 607-12.

25. McEllin B, Camacho CV, Mukherjee B, Hahm B, Tomimatsu N, Bachoo RM et al. PTEN loss compromises homologous recombination repair in astrocytes: implications for glioblastoma therapy with temozolomide or poly(ADP-ribose) polymerase inhibitors. Cancer Res 2010;70: 5457-64.

26. Ahmad M, Torky A, Glahn F, Scheubel RJ, Foth H. PARP-1 expression and activity in primary human lung cells. Arch Toxicol 2011;85:669-79. 